\title{
A HIGHLY SENSITIVE MICROSYSTEM BASED ON NANOMECHANICAL BIOSENSORS FOR GENOMICS APPLICATIONS
}

\author{
L.M. Lechuga $^{1^{*}}$, J.Tamayo ${ }^{1}$, M. Álvarez ${ }^{1}$, L.G. Carrascosa ${ }^{1}$, A. Yufera ${ }^{1}$, A. Rueda ${ }^{1}, J^{A}$ A. \\ Plaza ${ }^{1}$, K. Zinoviev ${ }^{1}$ and C Domínguez ${ }^{1}$, A. Zaballos ${ }^{2}$, M. Moreno and C. Martínez-A ${ }^{2}$, D. \\ Wenn $^{3}$, V. Bardinal, T. Camps, and C. Fontaine ${ }^{4}$, V. Díaz and A. Bernad. ${ }^{5}$ \\ ${ }^{1}$ Microelectronics National Center (CNM). CSIC \\ Isaac Newton 8, Tres Cantos, 28760 (Spain) \\ laura@imm.cnm.csic.es Tel: +34 918060700, Fax: +34 918060701 \\ ${ }^{2}$ Biotechnology National Center (CNB). CSIC. Spain. \\ ${ }^{3}$ ECS, University of Southampton, UK \\ ${ }^{4}$ LAAS-CNRS, France \\ ${ }^{5}$ GENETRIX, S.L
}

\begin{abstract}
A new DNA biosensor microsystem based on nanomechanical transducers is being developed. The microsystem comprises an array of 20 cantilevers, a microfludic system for delivery of the samples, an array of 20 lasers (VSCELs) and chips with the photodetectors and the CMOS circuitry for signal acquisition and conditioning. Robust immobilization procedures for the oligonucleotides receptor sequences has been also developed.
\end{abstract}

Keywords: microsystem, Si technology biosensor, genomics

There is an increasing interest in system based on micro/nanotechnologies for ultrasensitive and miniaturised biosensors and biochips. The application of this new micro- and nanotechnology techniques to microarray production will result in both miniaturization of biochip format and increase of the sensitivity of the assays performed. Biosensors based on Nanomechanics can specifically detect single-base mismatches in oligonucleotide hybridisation [1]. In these assays (see Figure 1), nucleic acids are immobilized on a side of a micromachined lever (active side). Exposure of the cantilever to a sample containing complementary nucleic acid gives rise to a cantilever bending (deflection) of a few nanometers. The nanomechanical response is due to the surface stress change of the active side with respect to the other side, in which DNA is not immobilized [2]. Surface stress arises from the electrostatic, van der Waals and steric intermolecular interactions on the surface. The deflection is measured with subnanometer resolution by an optical system (similar to that of an AFM), in which a laser beam reflects off the back of the cantilever to a position sensitive photodetector.

In the framework of an European project, (OPTONANOGEN) [3] we are developing a portable multibiosensor microsystem able to detect hybridization of nucleic acids partly complementary to human genes with femtomolar sensitivity and with the ability to discern single base variations. The biosensor microsystem consists of: i) an array of twenty microcantilevers as biosensing tranducers, ii) an optical detection system (lasers and photodetectors) capable of measuring the cantilever movement with sub-nanometer resolution and iii) a polymer microfluidic system for reagents delivery to the biosensor.

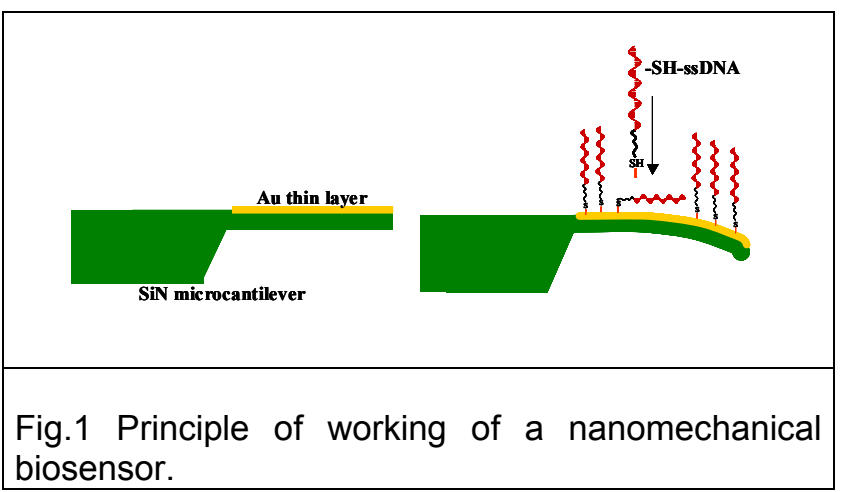

The main tasks done in order to obtain the biosensor microsystem are the following:

1. Fabrication of arrays of 20 microcantilevers. Normally, micrometer-sized cantilevers are designed for atomic force microscopy. But for application as highly sensitive biosensors the cantilevers have to be redesigned carefully according with the dimensions and the mechanical material properties. The cantilever geometry, dimensions and materials have been modelled in order to obtain a highly sensitive nanomechanical response and a small sensor area. A technology of fabrication of single-crystalline-silicon thin cantilevers using commercially available Silicon-On-Insulator (SOI) wafers has been developed and optimised to achieve 100\% 
yield of cantilevers per wafer. The technology allows the fabrication of arrays composed of 20 cantilevers, each being 200 $\mu \mathrm{m}$ long, $40 \mu \mathrm{m}$ wide, and $340 \mathrm{~nm}$ thick (see Figure 2).

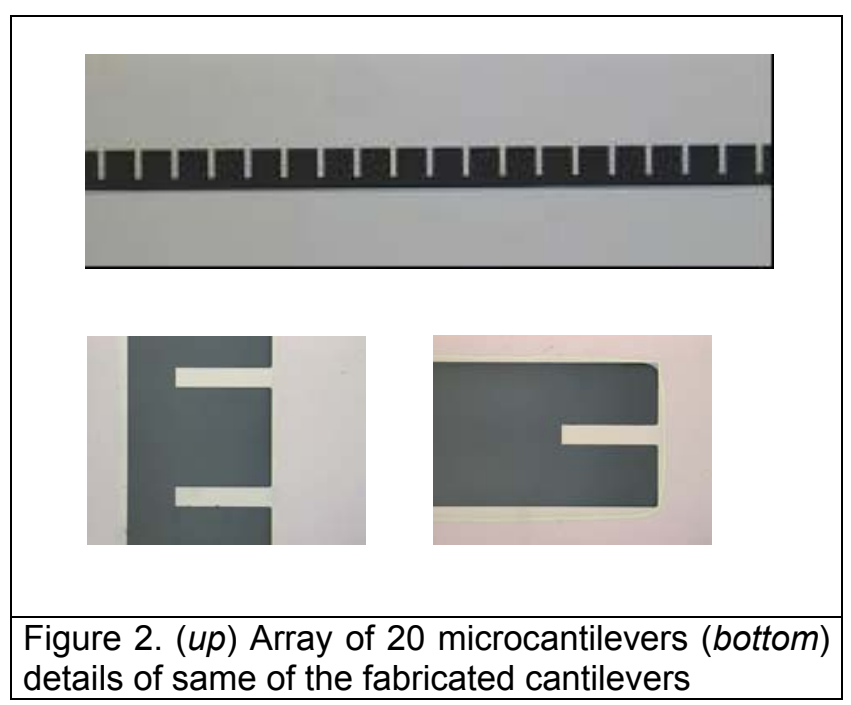

2. Development of a self-assembly chemistry which allows the addressing of DNA immobilization at the nanometer-scale level only on one side of the cantilever array. The optimised immobilisation procedure is based on thiolated self-assembled monolayers. Figure 3 shows the cantilevers' bending observed during the immobilisation of the oligonucleotide receptors.

3. A polymer microfluidic header has been developed and integrated with the cantilever array. The microfluidic header incorporates flow channels to allow the delivery of reagents to the cantilevers, and subsequent removal of waste. Two different types of flow cells have been implemented: (i) a common flow cell for the whole array (one channel) (ii) a discrete flow cell for independent measurement of each cantilever in the array (twenty channels).

4. Development of an optical detection subsystem which follows the parallelism of the cantilevers and the microfluidics subunits. A monolithic array of 20 VCSELs (Vertical Cavity Surface Emission Lasers) (emitting at $850 \mathrm{~nm}$ ) have been fabricated by MOCVD, characterised and integrated into the final packaging.

5. For the detection of the displacement of the laser beam reflected off the cantilever backside, an array of 20 segmented photodetectors, integrated with their respective amplifier and signal CMOS processing circuits have been designed and fabricated using IC silicon technology.
6. The final integration will be performed by incorporating the microfluidic header into a package that integrates the optical source, sensor array, photodetectors and CMOS control electronics.

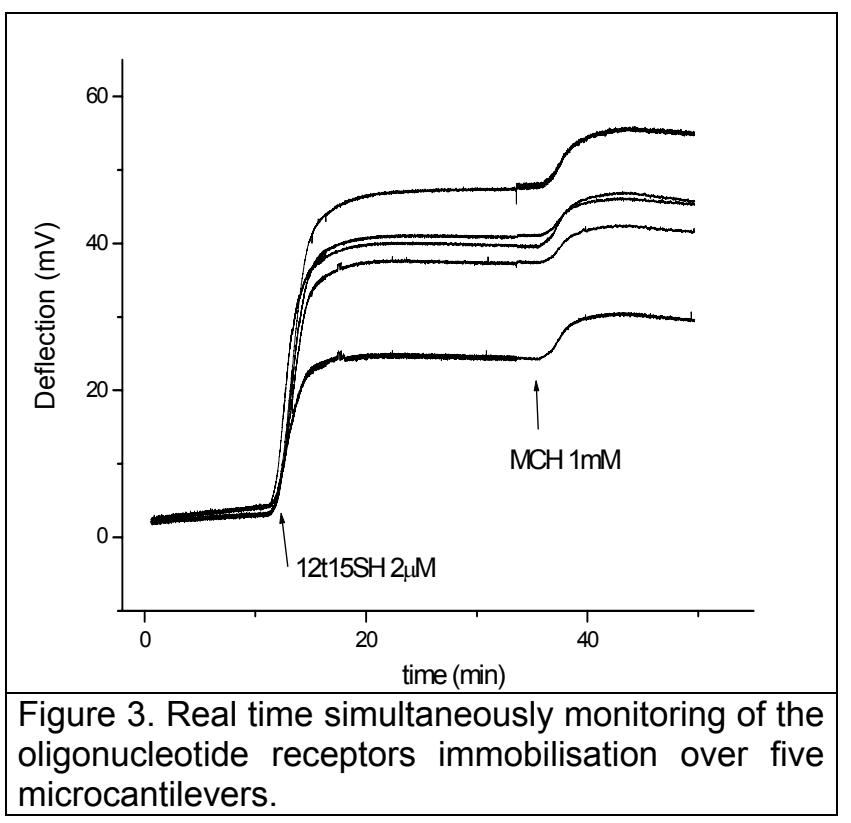

\section{ACKNOWLEDGEMENTS}

This work is being supported by the EU Contract IST-2001-37239.

\section{REFERENCES}

1. Fritz, M. K. Baller, H. P. Lang, H. Rothuizen, P. Vettiger, E. Meyer, H. J. Güntherodt, Ch. Gerber, J. W. Gimzewski, Science 288, 316 (2000)

2. M. Álvarez, A. Calle, J. Tamayo, A. Abad, A. Montoya and L.M. Lechuga. Biosensors \&

Bioelectronics 18, 649 (2003)

3. www.optonanogen.com 